\title{
Advanced Imaging of Pediatric Radial and Axillary Nerve Anatomy for Intraoperative Use
}

\author{
Jessica Koshinski, BS, Mark Mandel, BS, Max Cornell, BS, and Mark Seeley, MD \\ Department of Orthopaedic Surgery, Geisinger Medical Center, Danville, Pennsylvania, USA
}

\begin{abstract}
Introduction: Lateral percutaneous pin placement, intramedullary screw placement, and plate placement during surgical fixation of distal humeral fractures all risk iatrogenic injury to the radial nerve as it crosses from the posterior to anterior compartment at the level of the mid-humeral shaft. Current literature describing the "safe zone" to avoid damaging the radial nerve at this location focuses on adult vascular anatomy. Radial nerve anatomy changes throughout childhood, and determination of age specific safe zones will help minimize iatrogenic nerve palsy in the pediatric population. Axillary nerve anatomy was also assessed.
\end{abstract}

Methods: Electronic medical records were queried for humeral and shoulder MRIs that included the proximal humeral physis and the lateral epicondyle to assess the radial nerve or the lateral edge of the acromion and the upper $1 / 3$ of the humeral diaphysis to assess the axillary nerve.

Results/Discussion: Our study suggests the axillary nerve reaches its adult location around 14 years of age while the radial nerve does not reach its approximate adult location until 16 years of age. Before these ages extra precaution should be taken during surgical dissection and fracture fixation.

Keywords

Pediatric orthopedics, Radial nerve, Axillary nerve, MRI, Surgical safe zone

\section{Introduction}

In the pediatric population, humerus fractures are common and may be severe enough to require surgical repair and/or reconstruction. Unfortunately, there is a lack of research identifying surgical "safe zones" to avoid iatrogenic injury to important neurovascular structures traversing over the proximal and mid shaft of the humerus in the pediatric population. Adult anatomical mapping does not take the proportionally smaller limb size or effect of skeletal growth into account. The evolving anatomical location of the axillary and radial nerves over the proximal and mid humeral shaft of the aging child are of particular concern as lateral approaches to the proximal humerus and shoulder put terminal branches of the axillary nerve at risk [1]. Similarly, lateral percutaneous pin placement, intramedullary screw placement, and proximal extension of lateral plate placement during surgical fixation of supracondylar humerus fractures all risk iatrogenic injury to the radial nerve as it exits the spiral groove and crosses from the posterior to anterior muscular compartment [2].

We have chosen to examine the radial nerve at the vulnerable point as it exits the spiral groove and transitions from the posterior to anterior muscular compartment, and we have decided to examine the axillary nerve as it hugs the lateral aspect of the proximal humeral shaft just after it traverses from medial to lateral along the posterior aspect of the surgical neck. The purpose of this study is to use magnetic resonance imaging (MRI) in order to gain a more thorough understanding of the maturing locations of the axillary and radial nerves in growing children.

\section{Methods}

This was a retrospective imaging review of electronic medical records that were collected from a tertiary hospital's patient database. Patient records of male and female pediatric patients ranging from 0 to 18 -years-old were queried for humeral MRIs that included the proximal humeral physis and the lateral epicondyle and/or the lateral edge of the acromion down to the mid humeral shaft. Patients were required to have an axial and coronal T1-weighted MRI of one of the

*Corresponding author: Mark A Seeley, Geisinger Medical Center, Danville, PA, 100 N Academy Avenue, 17821, USA

Accepted: July 02, 2021

Published online: July 04, 2021

Citation: Koshinski J, Mandel M, Cornell M, et al. (2021) Advanced Imaging of Pediatric Radial and Axillary Nerve Anatomy for Intraoperative Use. Adv Tech Musculoskelet Surg 6(1):66-69 
Citation: Koshinski J, Mandel M, Cornell M, et al. (2021) Advanced Imaging of Pediatric Radial and Axillary Nerve Anatomy for Intraoperative Use. Adv Tech Musculoskelet Surg 6(1):66-69

above specified regions. Patients with pathology that altered the anatomical structure of this area were excluded. The point at which the radial nerve transitions from the posterior compartment to the anterior compartment of the arm was identified on axial imaging. This point was cross-referenced with the corresponding coronal image and measurements from this point to the lateral epicondyle were recorded. Similarly, the axillary nerve was traced as it transitions across the posterior aspect of the humerus along the posteromedial region of the surgical neck. We marked the nerve on axial imaging at the exact point at which it becomes visible again on the lateral aspect of humerus in the axial view. This point was cross-referenced with the corresponding coronal image, and measurements from this point to the lateral edge of the acromium were recorded (Figure 1 and Figure 2).

\section{Results}

In total, we identified $53 \mathrm{MRI}$ 's that fit our inclusion criteria with an age range of 0.23-17 years-old. Females represented $56 \%$ of patients $(n=25)$ and males represented $44 \%$ of patients $(n=19)$. The most common reason for imaging was investigation of suspected lytic lesion $(n=6)$ followed by suspected subluxation ( $n=4)$. Again, pathology obscuring normal anatomy (e.g. traumatic brachial plexus injury or large aneurysmal bone cyst).

A total of 43 unique patients MRI's were measured for axillary nerve distance. Measurements were determined and grouped by age range 0-3 years-old $(n=6), 4-6$ years-old ( $n$ $=4), 7-9$ years-old $(n=4), 10-13$ years-old $(n=10)$, and $14+$ years-old $(n=19)$. Mean distance from the lateral edge of

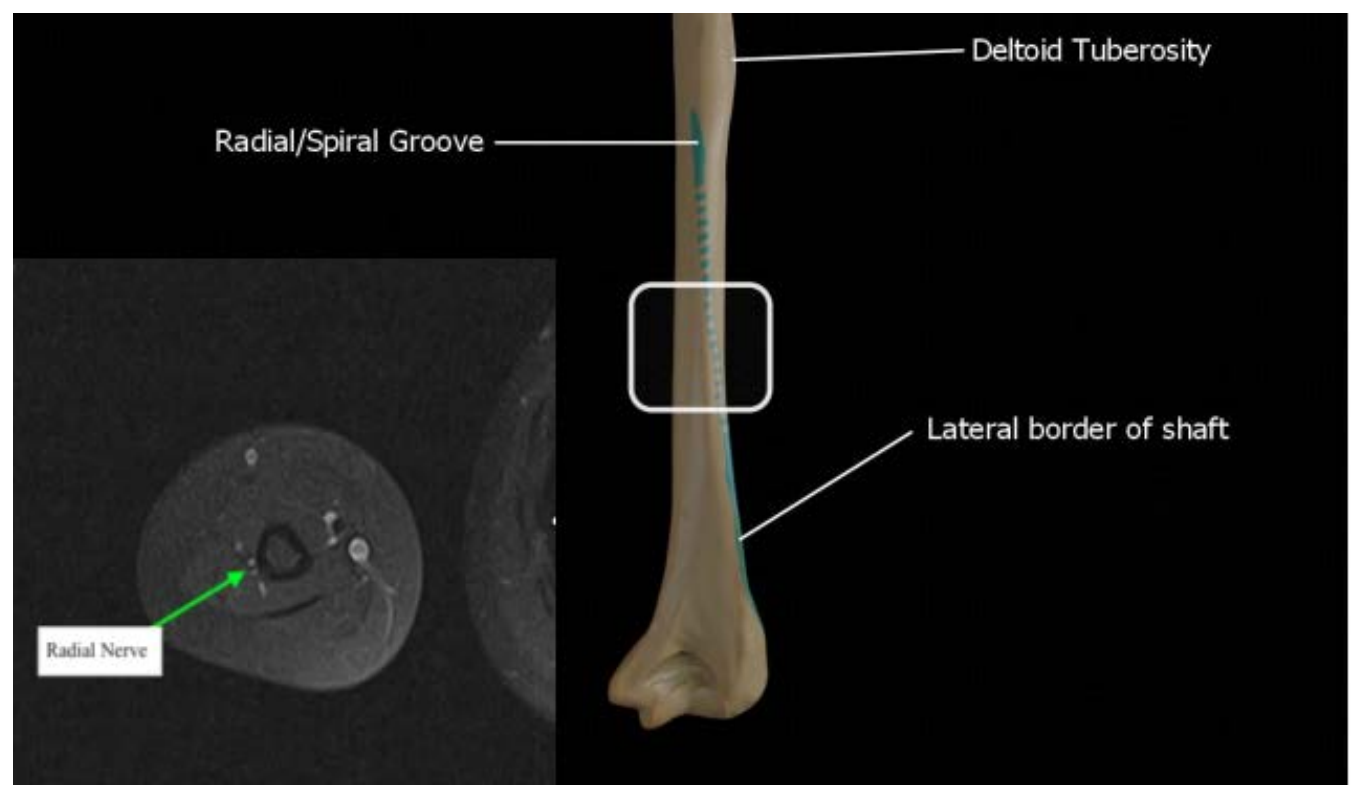

Figure 1: Location of radial nerve as it exits the spiral grooves and begins its transition from the posterior to anterior muscular compartment (photograph by Doctor Jana, distributed under the Creative Commons Attribution-Share Alike 4.0 International license).

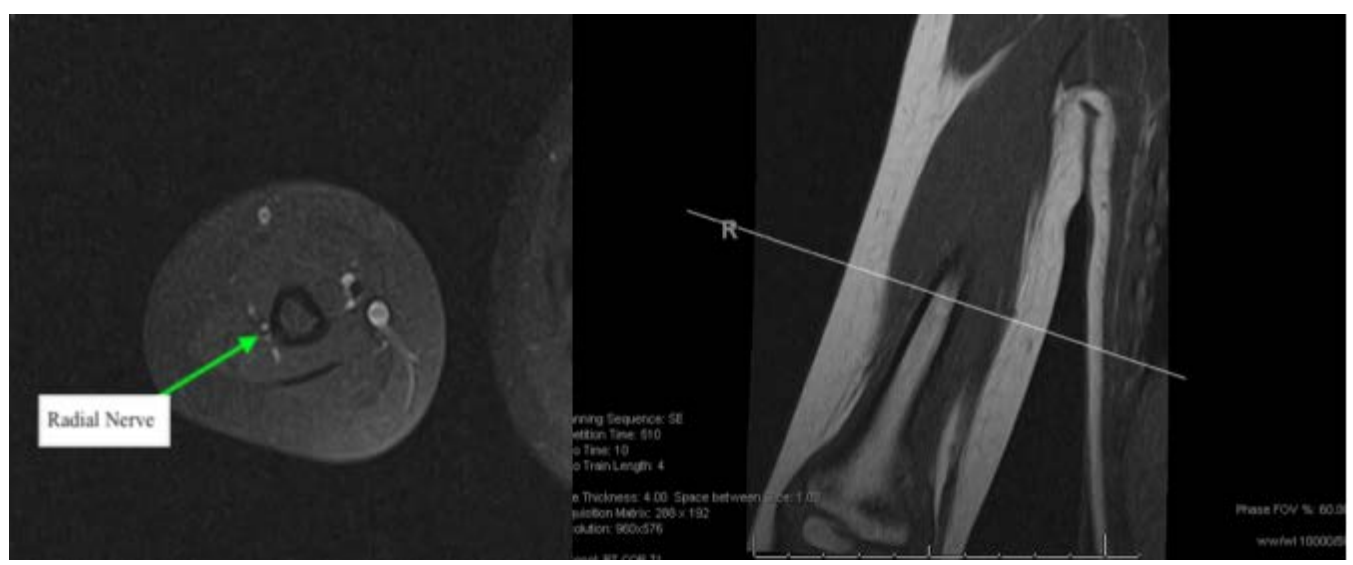

Figure 2: Nerve location identified on axial imaging (left). Imaging software was utilized to find corresponding coronal location (right). Measurement from lateral epicondyle to nerve location was measured in a straight line on the coronal image. 
Citation: Koshinski J, Mandel M, Cornell M, et al. (2021) Advanced Imaging of Pediatric Radial and Axillary Nerve Anatomy for Intraoperative Use. Adv Tech Musculoskelet Surg 6(1):66-69

the acromium to the point at which the axillary nerve at the previously described location were reported as: 0-3 years-old distance $=30.72 \mathrm{~mm}$ (range, 24.7-33.1 $\mathrm{mm}$ ); 4-6 years-old distance $=36.40 \mathrm{~mm}$ (range, 33.1-40.3 $\mathrm{mm}) ; 7-9$ years-old distance $=48.28 \mathrm{~mm}$ (range, $45.9-49.9 \mathrm{~mm}) ; 10-13$ years-old and older $=55.58 \mathrm{~mm}$ (range, $50.4-59.1 \mathrm{~mm}$ ), and 14-yearsold and older $=63.22 \mathrm{~mm}$ (range, 60-69 mm) (Figure 3).

A total of 10 unique patients MRI's were measured for radial nerve distance. Measurements were determined and grouped by age range 0-3 years-old $(n=1), 4-6$ years-old ( $n$ $=2$ ), 7-8 years-old $(n=2), 11-15$ years-old $(n=3)$, and $16+$ years-old $(n=2)$. Mean distance from the lateral epicondyle to the point at which the radial nerve crosses from the posterior to anterior compartment of the arm were reported as: 0-3 years-old distance $=27.5 \mathrm{~mm}$; $4-6$ years-old distance $=49.75 \mathrm{~mm}$ (range, $47.7-51.8 \mathrm{~mm}$ ) ; 7-8 years-old distance $=$ $57.85 \mathrm{~mm}$ (range, $57.8-57.9 \mathrm{~mm}$ ); 11-years-old and older = $89.78 \mathrm{~mm}$ (range, 83.5-97 mm) (Figure 4).

\section{Discussion}

Due to the lack of defined pediatric specific "safe zones" for avoiding the axillary nerve during surgery, we set out to further map the maturing location of the nerve and determine the age at which adult location guidelines become practical. The axillary nerve runs obliquely in front of the subscapularis muscle, crossing its inferior border, then to the posteromedial portion of the surgical neck of the humerus in the quadrilateral space before entering the deltoid from the posterolateral aspect of the humerus and branching into terminal sensory and motor fibers [3]. T1 weighted MRI imaging enabled us to identify the axillary nerve directly after it crossed posterior to the surgical neck and hugs the posterolateral aspect of the humeral shaft before splitting into motor and sensory branches within the deltoid. We measured the distance between this point and the lateral edge of the acromion due to its utility as a surgical landmark. Our results indicated that there is a near-linear relationship in distance between these two points as age increases. Importantly, children do not reach the adult safe zone estimate of 6-7 cm distal to the lateral edge of the acromion [4] until they are 14-years-old (Figure 5).

The radial nerve plays a crucial role in innervation of the posterior compartment of the arm, and its path has been well-established in the adult population [5]. Originating from the posterior cord of the brachial plexus, the radial nerve exits the axillary region and courses through the upper arm deep to the triceps brachii, on the posterior side of the humerus, in the radial groove, traversing medial to lateral, and accompanied by the deep brachial artery [6]. It enters the forearm anterior to the lateral epicondyle, traveling through the cubital fossa and terminating as deep and superficial branches [7]. In adults, several anatomical landmarks are used to reduce the risk of radial nerve injury in surgical procedures for interventions of the distal humerus and elbow $[2,8,9]$.

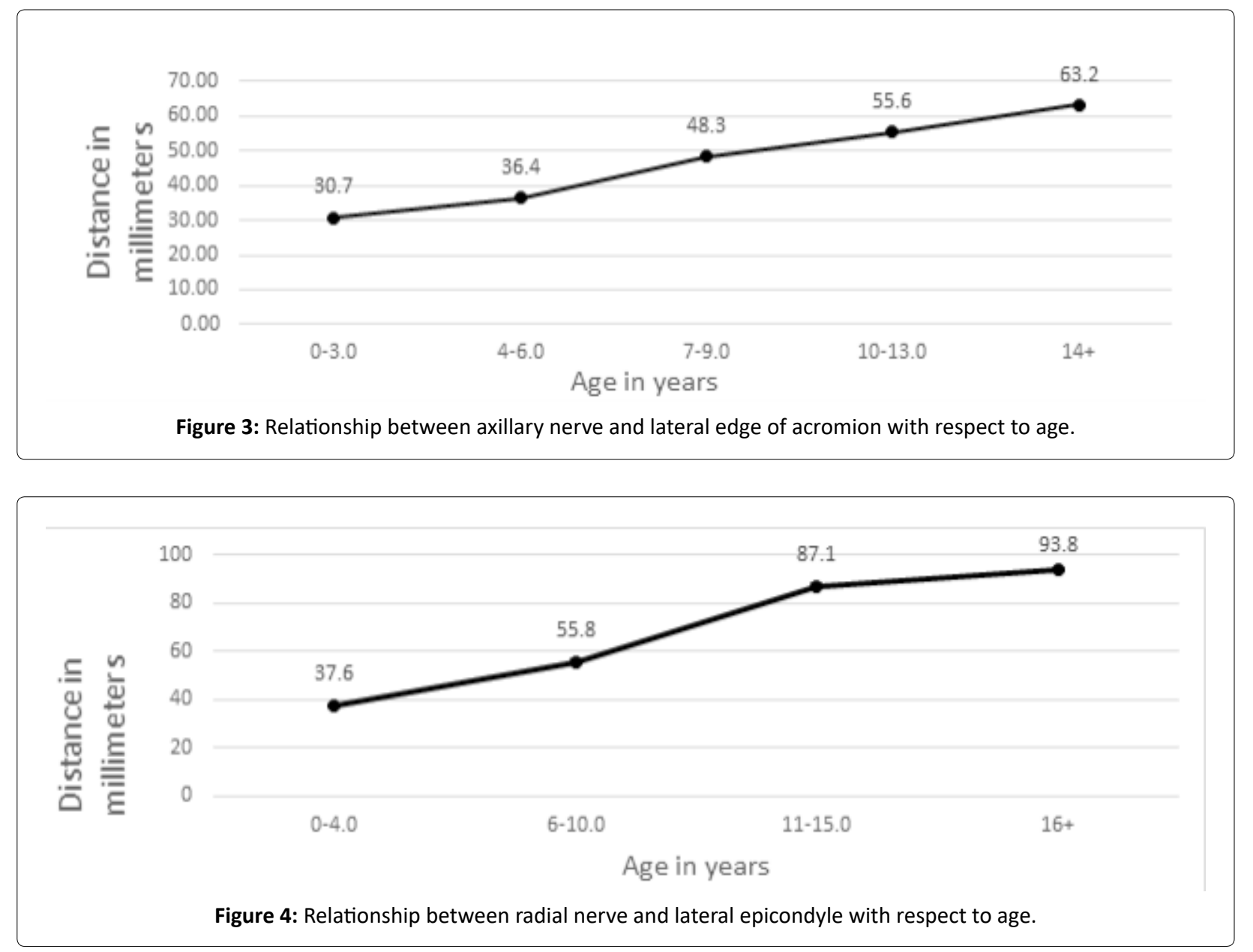




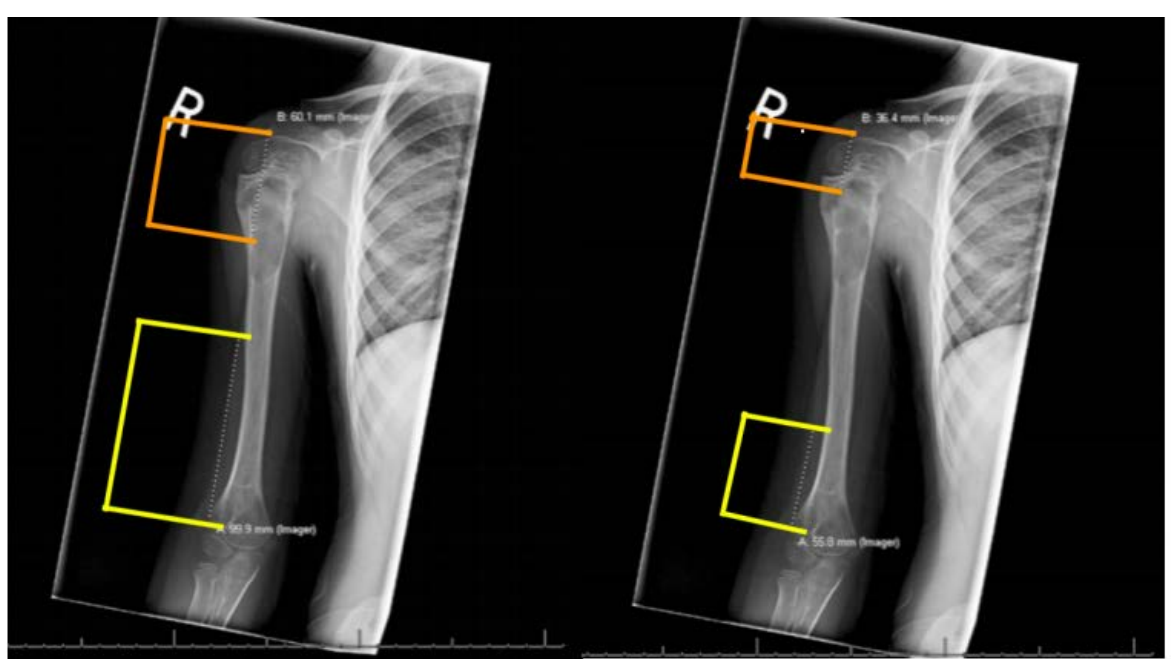

Figure 5: Images of a 5-year-old male with traditional adult nerve locations marked (left radiograph) $6 \mathrm{~cm}$ distal to lateral edge of acromium for axillary nerve (orange) and $10 \mathrm{~cm}$ proximal to lateral epicondyle for radial nerve (yellow). The radiograph on the right represents correct age matched locations of said nerves.

Using the lateral epicondyle as our landmark we measured the distance to the radial nerve as it exits the spiral groove and transitions from the posterior to anterior muscular compartment. At this point the radial nerve passes in close proximity to the humerus which poses risk for iatrogenic injury during proximal extension of posterolateral approaches to the elbow, percutaneous pin placement, and fracture plating [9]. Our results again showed a near-linear increase in measured distance between the spiral groove and lateral epicondyle as age increased however, the adult "safe zone" estimation of remaining $9-10 \mathrm{~cm}$ proximal to the lateral epicondyle [2] is not valid until the child reaches 16-years-old.

Limitations of this study included a small sample size of 53 MRI's with 10 MRI's of the radial nerve and 43 MRI's of the axillary nerve. Imaging in the proximal and mid shaft of the humerus required for this study is generally reserved for anatomy altering pathology such as tumors, cysts, and neurovascular injury such as brachial plexus injury. Thus, the retrospective aspect of our study contributed to our low sample size. Another limitation to our sample size was the requirement for unambiguous imaging showing the nerves at our pre-determined points of interest.

The strength of our study is illustrated in that our findings are aligned with the limited existing, research conducted on this topic. In 2018, Nielsen, et al. analyzed radial nerve location at the same point of interest in 21 pediatric MRI images [10]. Their study established a relative safe zone of $6 \mathrm{~cm}$ proximal to the distal humeral physis for all children ages 6-yearsold or above. While our study identified several 6-year-old patients just below the $6 \mathrm{~cm}$ measurement, our results are comparable, and show adult nerve location estimates are not accurate in a large proportion of pediatric patients.

In conclusion, our research has demonstrated that the "safe zones" for adults do not translate into useful data for pediatric patients. In fact, we found that the "safe zones" for adults may not be useful until the child turns 16-yearsold regarding the radial nerve and 14-years-old regarding the axillary nerve. Coordination of larger, higher power studies to define specific pediatric "safe zones" with respect to height, weight, sex, and other patient characteristics would benefit the field.

\section{References}

1. Perlmutter GS (1999) Axillary nerve injury. Clin Orthop Relat Res 368: 28-36.

2. Cheung EV, Steinmann SP (2009) Surgical approaches to the elbow. Journal of the American Academy of Orthopedic Surgeons 17: 325-333.

3. Tessler J, Talati R (2021) Axillary nerve injury In: StatPearls [Internet]. Treasure Island (FL): StatPearls Publishing; 2021 Jan 2021 Apr 17.

4. Patra A, Singh M, Kaur H, et al. (2018) Re-definition of position and calculation of safe area for axillary nerve in deltoid muscle with its clinical relevance: A cadaveric study. Anat Cell Biol 51: 93-97.

5. Eglseder WA (2012) Distal humeral fractures: Impact of lateral approach and fracture-specific plating on radial nerve palsies. Techniques in Hand \& Upper Extremity Surgery 16: 127-131.

6. Glover NM, Murphy PB (2021) Anatomy, shoulder and upper limb, radial nerve. [Updated 2020 Jul 31]. In: StatPearls [Internet]. Treasure Island (FL): StatPearls Publishing; 2021 Jan.

7. Ljungquist KL, Martineau P, Allan C, et al. (2014) Radial nerve injuries. J Hand Surg Am 40: 166-172.

8. Jawa A, McCarty P, Doornberg J, et al. (2006) Extra-articular distal-third diaphyseal fractures of the humerus: A comparison of functional bracing and plate fixation. J Bone Joint Surg Am 88: 2343-2347.

9. Kamineni S, Ankem H, Patten DK, et al. (2009) Anatomic relationship of the radial nerve to the elbow joint: Clinical implications of safe pin placement. Clin Anat 22: 684-688.

10. Nielsen E, Andras LM, Skaggs DL, et al. (2018) Quantifying the location of the radial nerve in children for intraoperative use. $J$ Pediatr Orthop 38: 292-295. 